\title{
Materiali e tecniche costruttive del Mugello tra basso Medioevo e prima Età Moderna
}

\section{Materials and building techniques in Mugello from the Late Middle Ages to the Early Modern Age}

\author{
Andrea Arrighetti \\ Dipartimento di Scienze Storiche e dei Beni Culturali - Università degli Studi di Siena \\ andrea.arrighetti@unisi.it
}

\begin{abstract}
RIASSUNTO
Il Mugello è un'area a medio-alto rischio sismico situata sulla catena montuosa appenninica a confine fra la Toscana e I'Emilia Romagna. Il territorio si caratterizza per una nutrita presenza di insediamenti di lunga durata caratterizzata da edifici storici ben conservati, dei quali la maggior parte risultano strutture religiose. Una zona del Mugello, tra il 2010 ed il 2014, è stata interessata dal progetto "Archeologia dell'architettura e rischio sismico in Mugello", una ricerca incentrata sulla sperimentazione del potenziale informativo del processo di analisi archeologica come forma di conoscenza, prevenzione e tutela dell'edilizia medievale dal rischio sismico. Fra i risultati che sono emersi dalle indagini archeologiche hanno svolto un ruolo centrale le considerazioni inerenti I'approvvigionamento ed utilizzo dei materiali costruttivi per l'edificazione e la modifica delle strutture architettoniche, in un periodo compreso fra il Basso Medioevo e l'Età Moderna.
\end{abstract}

Parole Chiave: Archeosismologia; Architettura; Tecniche Costruttive; Cronotipologia.

\begin{abstract}
Mugello is a medium-high seismic risk area situated on the Italian Apennine mountain range, between Tuscany and Emilia Romagna. The territory is characterized by a large presence of long duration settlements characterized by well-preserved historic buildings, most of which are religious' architectonical complexes. An area of Mugello, between 2010 and 2014, was characterized by the project "Archaeology of Buildings and seismic risk in Mugello", a research focused on testing the potential information of the process of archaeological analysis of buildings as a form of knowledge, prevention and protection of medieval seismic risk settlements. Among the results that have emerged from the archaeoseismological investigation have played a central role the considerations pertaining to the supplying and use of building materials for the construction and modification of architectural structures, in a period between the late Middle Ages and the Modern Age.
\end{abstract}

Keywords: Archaeoseismology; Architecture; Building's techniques; Chronotipology.

Recibido: 01-04-2015. Aceptado: 08-02-2016.

Arrighetti, A. 2016: "Materiali e tecniche costruttive del Mugello tra basso Medioevo e prima Età Moderna", Arqueología de la Arquitectura, 13: e037. doi: http://dx.doi.org/10.3989/arq.arqt.2016.001

\section{Copyright}

(C) 2016 CSIC. This is an open-access article distributed under the terms of the Creative Commons Attribution (CC-by) Spain 3.0 License. 


\section{INTRODUZIONE}

Il Mugello è un'area a medio-alto rischio sismico situata sulla catena montuosa appenninica a confine fra la Toscana e l'Emilia Romagna. Il territorio si caratterizza per una nutrita presenza di insediamenti di lunga durata (le maggiori attestazioni si collocano cronologicamente intorno ai secoli centrali del Medioevo e presentano una continuità insediativa fino ai giorni nostri) caratterizzata da edifici storici ben conservati, dei quali la maggior parte risultano strutture religiose.

Una zona del Mugello compresa fra i comuni di Scarperia, Borgo San Lorenzo, Vicchio e Barberino, tra il novembre 2010 ed il marzo 2014, è stata interessata dal progetto "Archeologia dell'architettura e rischio sismico in Mugello", una ricerca incentrata sulla sperimentazione del potenziale informativo del processo di analisi archeologica come forma di conoscenza, prevenzione e tutela dell'edilizia medievale dal rischio sismico ${ }^{1}$. Fra i risultati che sono emersi dalle indagini archeologiche hanno svolto un ruolo centrale le considerazioni inerenti l'approvvigionamento ed utilizzo dei materiali costruttivi per l'edificazione e la modifica delle strutture architettoniche, in un periodo compreso fra il Basso Medioevo e l'Età Moderna.

Uno degli aspetti più interessanti dell'analisi della storia costruttiva degli edifici storici è stata la possibilità di individuare, catalogare ed in alcuni casi datare i sistemi costruttivi che li caratterizzano, utilizzando i dati elaborati per la ricostruzione degli aspetti storici legati al territorio e per la definizioni di elementi cronologici specifici in funzione della proposizione di crono-tipologie di riferimento. Le fonti utilizzate nella ricerca sono state essenzialmente due: da un lato le fonti storiche, utili per fornire datazioni su specifiche vicende costruttive sia in cronologia relativa che assoluta, e dall'altro la lettura stratigrafica dei paramenti a vista, che invece porta a ricostruire un'evoluzione delle fasi

\footnotetext{
Il progetto è convogliato nella tesi di dottorato “Archeologia dell'Architettura e rischio sismico in Mugello. L'apporto della stratigrafia alla conoscenza dei terremoti storici", discussa dall'autore nel marzo 2014 presso l'Università degli Studi dell'Aquila ed inserita nel XXVI ciclo del Dottorato di Ricerca in Archeologia Medievale. La realizzazione del lavoro ha previsto il confronto con numerosi docenti e professionisti che hanno migliorato ed implementato il progetto in base al settore disciplinare di loro appartenenza. In particolare il confronto più articolato è avvenuto con: il Prof. Guido Vannini dell'Università degli Studi di Firenze (tutor del dottorato), il Prof. Fabio Redi dell'Università degli Studi dell'Aquila (responsabile scientifico del Dottorato di Ricerca), il Prof. Roberto Parenti dell’Università degli Studi di Siena, il Prof. Francesco Doglioni dell'IUAV di Venezia, il Prof. Paolo Peduto dell'Università degli Studi di Salerno, il Prof. Gerrit Jasper Schenk dell'Università di Darmstadt. La parte del progetto relativa alla metodologia archeosismologica di analisi dell'architettura è confluita nella monografia "L'archeosismologia in architettura. Per un manuale" edita dalla casa editrice Firenze University Press (Arrighetti 2015).
}

di costruzione del manufatto analizzato, proponendo un terminus cronologico per le singole operazioni di costruzione-distruzione. Questo tipo di analisi dunque ha proposto scenari interessanti sia per la caratterizzazione e datazione delle operazioni antropiche di modifica dell'edificio, sia per quelle derivate da eventi naturali, come appunto i terremoti.

I dati ricavati, diversi per quantità e qualità di informazioni, hanno fornito elementi di notevole interesse riguardanti diversi aspetti della ricerca. La proposizione di crono-tipologie dei sistemi costruttivi ha permesso, ad esempio, di catalogare e datare le diverse murature presenti nel contesto analizzato che, una volta confrontate fra di loro, hanno offerto la possibilità di ipotizzare l'utilizzo di specifici espedienti nella loro costruzione. Allo stesso tempo la definizione di una crono-tipologia degli interventi di restauro in zona sismica ha portato ad ipotizzare il ruolo delle maestranze e delle committenze nelle fasi post-sisma. Questa prassi è risultata indispensabile per ipotizzare la presenza o l'assenza di una Cultura Sismica Locale (Pierotti-Ulivieri 2001; Pierotti 2003) nel knowhow dei costruttori presenti nella zona indagata.

\section{LINEE METODOLOGICHE DI ANALISI DEI MANUFATTI}

Il progetto "Archeologia dell'Architettura e rischio sismico in Mugello" è stato caratterizzato per una metodologia di analisi del contesto basata sull'approccio archeologico allo studio dei manufatti architettonici medievali presenti al suo interno. In generale ${ }^{2}$, dopo una prima ricognizione sistematica del contesto di studio, i cui confini sono ben visibili in fig.4, che ha portato all'identificazione di numerosi edifici da sottoporre ad analisi, e la conseguente scelta del campione più rappresentativo in termini di qualità delle strutture murarie a vista (che dunque permettessero una leggibilità stratigrafica delle fasi costruttive) e di rappresentatività per il periodo di interesse (dal XII al XX secolo), è stata operata una lettura stratigrafica di dodici complessi architettonici. L'analisi è stata condotta per gruppi di attività costruttive (fig.1), arrivando all'identificazione delle Unità Stratigrafiche Murarie (USM) solo dove si rendeva necessario approfondire il dettaglio della ricerca ${ }^{3}$.

\footnotetext{
2 Per approfondimenti sull'intero processo di analisi del contesto di studio e sulla lista delle fonti edite ed inedite studiate si rimanda a Arrighetti 2015.

3 L'approfondimento è stato eseguito solo dove si presentavano nodi stratigrafici particolarmente complessi o dove il dettaglio permetteva di elaborare dati indispensabili alla corretta interpretazione dell'evoluzione costruttiva del complesso architettonico.
} 


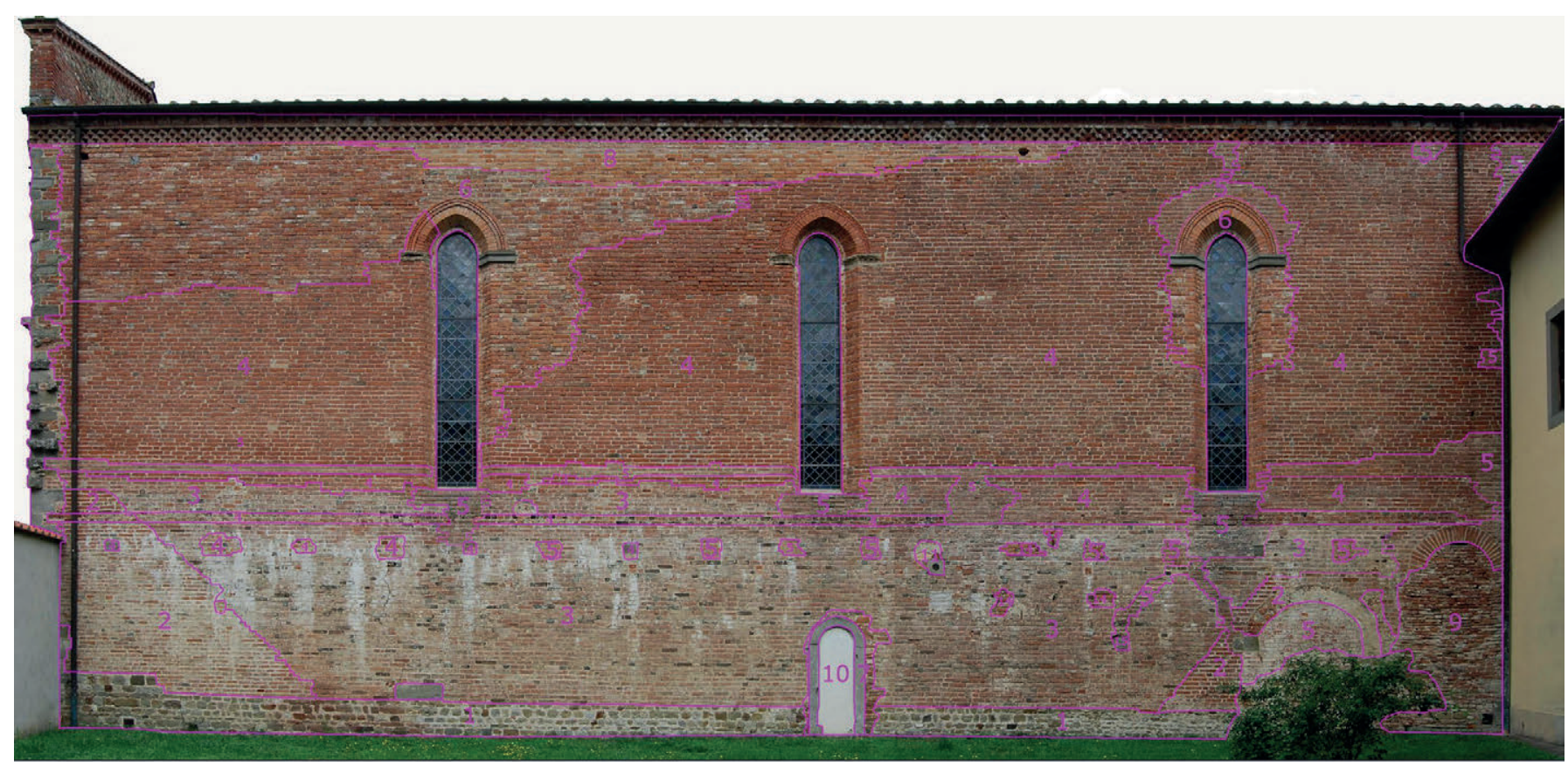

Fig. 1. L'immagine mostra una esemplificazione della lettura stratigrafica operata per gruppi di attività costruttive. In particolare la figura presenta I'analisi stratigrafica del prospetto sud della chiesa di San Francesco a Borgo San Lorenzo (FI).

All'analisi stratigrafica è dunque seguita la costruzione di una cronologia relativa fra le attività costruttive che ha portato ad una prima interpretazione del dato archeologico e ad una caratterizzazione crono-tipologica delle singole tecniche costruttive murarie presenti nelle strutture. A tutto ciò ha fatto seguito uno spoglio delle fonti scritte edite ed inedite, presenti in archivi e biblioteche locali e nazionali, che ha permesso di proporre cronologie assolute per alcune fasi. Il passo finale è stato l'interpretazione dei dati elaborati attraverso l'intero processo di analisi archeologica dei dodici manufatti, in scala territoriale, elaborando dunque risultati che permettessero di comprendere al meglio i cambiamenti nelle dinamiche insediative dell'area e gli aspetti sociali, economici e politici collegati ad essi.

\section{L'APPROVVIGIONAMENTO E L'UTILIZZO DEI MATERIALI DA COSTRUZIONE FRA BASSO MEDIOEVO E PRIMA ETA' MODERNA}

Il Mugello è morfologicamente definito da una zona centrale depressa, solcata nella sua parte meridionale dal fiume Sieve, lungo cui corre una stretta striscia alluvionale. Questa zona centrale è costituita da terrazzi fluvio lacustri. A nord si trova la catena dell'Appennino costituita prevalentemente da formazioni geologiche di composizione arenacea, a sud si trova la catena di Monte Giovi - Monte Senario, costituita da formazioni arenacee nella zona orientale e formazioni calcareo marnose (Pietra Alberese) nella zona occidentale.

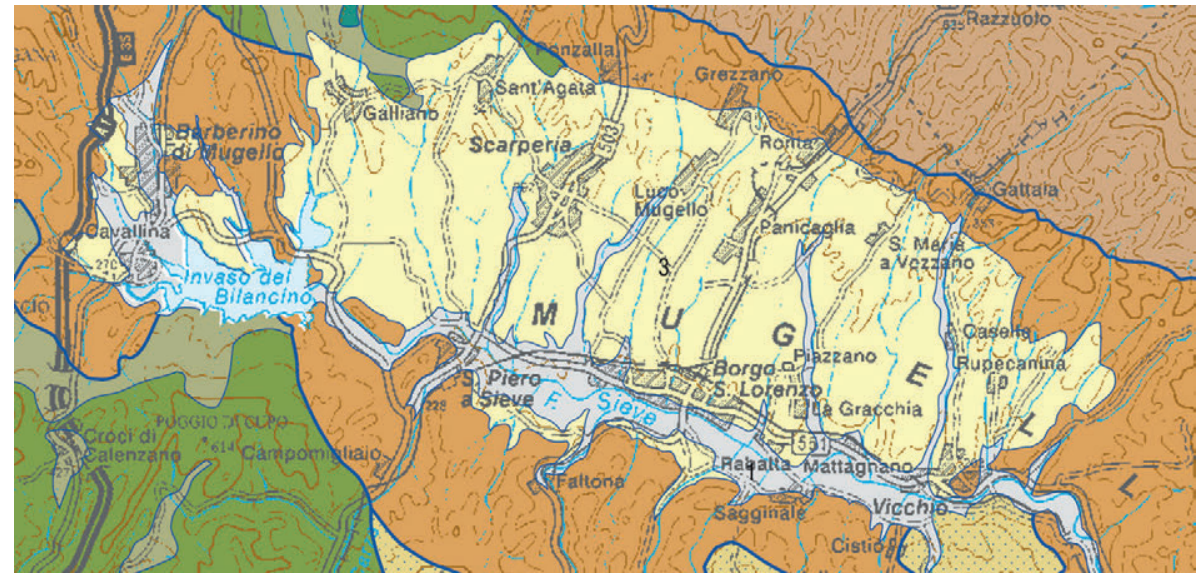

Fig. 2. Particolare relativo al Mugello della Carta Geologica della Toscana 


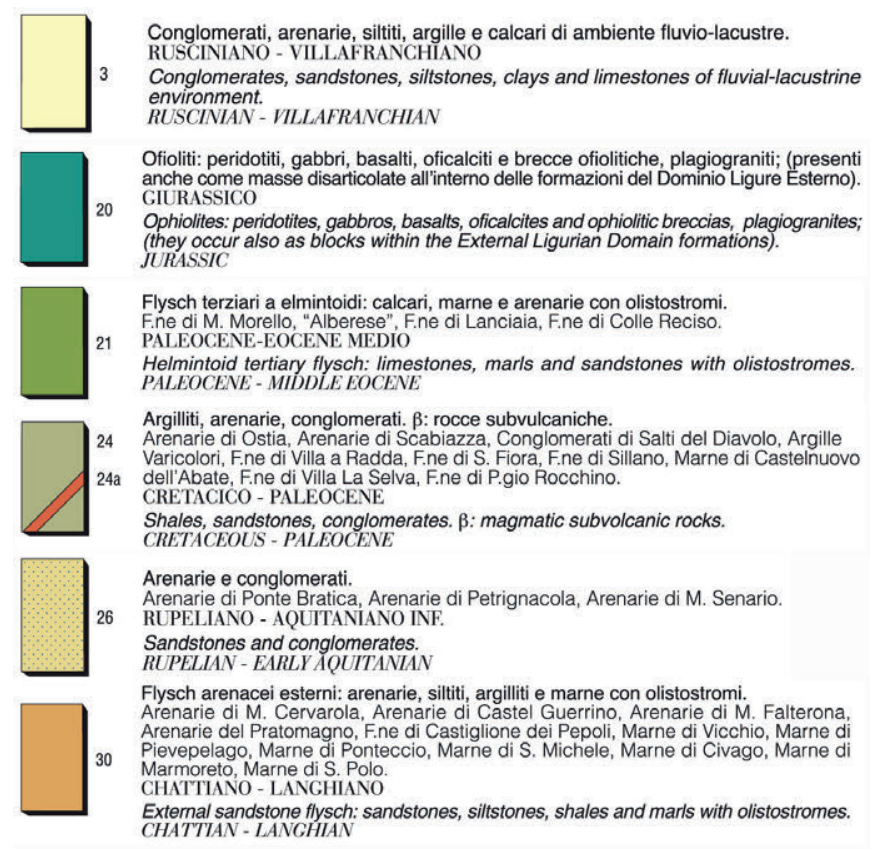

Fig. 3. Le materie prime affioranti nel contesto in esame

Mettendo in relazione la porzione della Carta Geologica della Regione Toscana, mostrata in fig.2, con i risultati ottenuti dall'analisi archeologica dei dodici edifici descritti nel paragrafo precedente, emergono dati di notevole interesse riguardanti l'impiego dei diversi materiali da costruzione in un arco cronologico che spazia dai secoli centrali del Medioevo alla prima Età Moderna.

In particolare gli edifici analizzati testimoniano l'utilizzo dei seguenti materiali:

1. Pieve di Sant'Agata del Mugello:

a) XII secolo: Pietra Alberese, Marmo Verde di Monte Calvi (elementi decorativi), Pietra Serena (materiale da costruzione e di reimpiego)

b) XIII - XIV secolo: Pietra Alberese, Marmo Verde di Monte Calvi (di reimpiego), Pietra Serena (di reimpiego)

c) XVI - inizi XVII secolo: Pietra Serena, Pietra della Formazione di Sillano (di reimpiego), Pietra Alberese (di reimpiego)

2. Pieve di San Giovanni Maggiore:

a) XI-XII secolo: Pietra Serena ed elementi in laterizio stracotti e vetrificati (elementi decorativi)

b) XIV secolo: Pietra Serena

c) XVI-XVII secolo: Pietra Serena e Laterizio

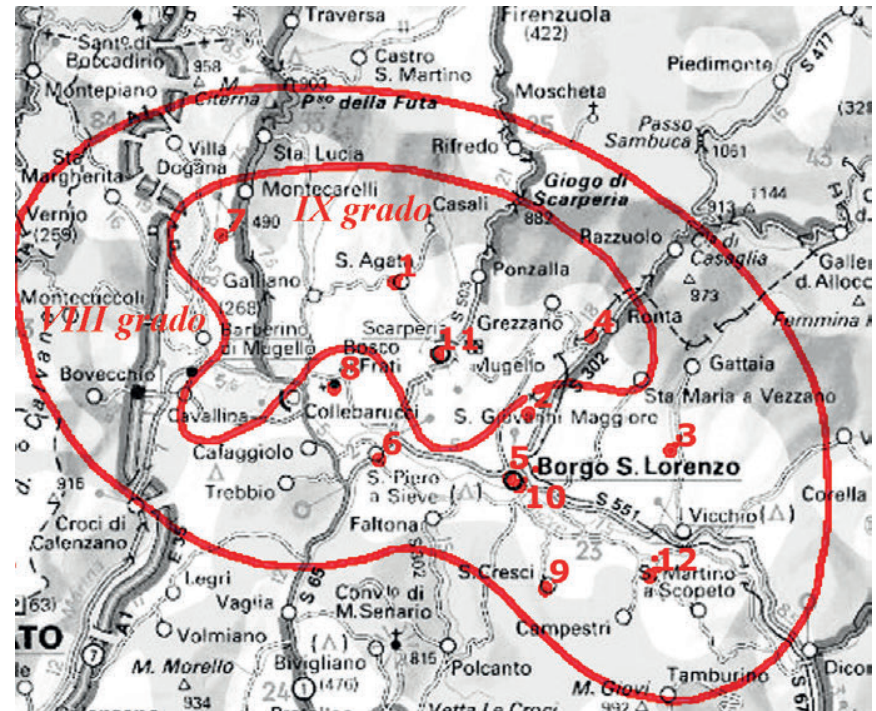

Fig. 4. I dodici edifici analizzati nel progetto "Archeologia dell'Architettura e rischio sismico in Mugello" e il confine del contesto di studio, quest'ultimo corrispondente all'VIII grado di Intensità Macrosismica ipotizzato dall'Istituto Nazionale di Geofisica e Vulcanologia per il terremoto del 13 giugno 1542.

3. Pieve di San Cassiano in Padule

a) X secolo: Pietra Serena

b) XVI-XVII secolo: Laterizio

4. Chiesa Vecchia di San Michele a Ronta
a) XIII secolo: Pietra Serena
b) XV secolo: Pietra Serena
c) XVI-XVII secolo: Pietra Serena e Laterizi

5. Pieve di San Lorenzo a Borgo

a) X-XI secolo: Pietra Alberese

b) Dal XIII secolo in poi: Laterizio

6. Pieve di San Pietro a San Piero a Sieve
a) X-XI secolo: Pietra Alberese
b) XIII-XIV secolo: Pietra Alberese e Formazione di Sillano
c) XV secolo: Pietra Alberese
d) Inizi XVII secolo: Pietra Arenaria e Materiale Misto

7. Pieve di San Gavino Adimari

a) X-XI secolo: Pietra Alberese

b) Metà XIII secolo: Pietra Alberese e Formazione di Sillano

c) XVI-XVII secolo: Laterizio e Pietra Serena 


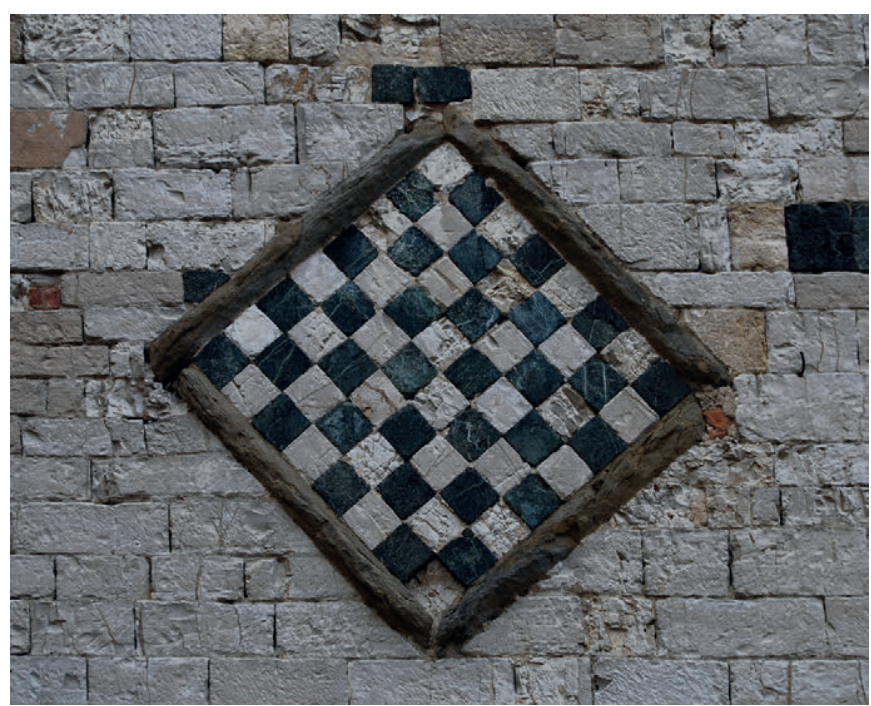

8. Chiesa di San Bonaventura del Bosco ai Frati
a) XI-XII secolo: Pietra Alberese
b) XIII-XIV secolo: Laterizio
c) XV secolo: Pietra Alberese
d) XVI-XVII secolo: Pietra Serena

9. Pieve di San Cresci in Valcava

a) X-XI secolo: Arenaria di Monte Senario

b) XIII-XIV secolo: Pietra Alberese e Arenaria di Monte Senario

10. Chiesa di San Francesco a Borgo
a) XI-XII secolo: Pietra Serena
b) Dal XIII secolo in poi: Laterizio

11. Palazzo dei Vicari di Scarperia
a) XIV secolo: Pietra Serena
b) XV secolo: Pietra Serena e Laterizio
c) XVI-XVII secolo: Pietra Serena

12. Chiesa di Santa Maria a Fabbrica

a) XI-XII secolo: Arenaria di Monte Senario

b) XVI-XVII secolo: Laterizio

I principali materiali da costruzione utilizzati nell'edilizia storica del Mugello appaiono dunque la Pietra Alberese (calcare marnoso), la Pietra Serena (arenaria), l'Arenaria di Monte Senario ed il laterizio. A questi si aggiungono il Marmo Verde di Monte Calvi (serpentinite, fig.5) e il laterizio stracotto e vetrificato (fig.6) utilizzati in modo molto sporadico come materiale

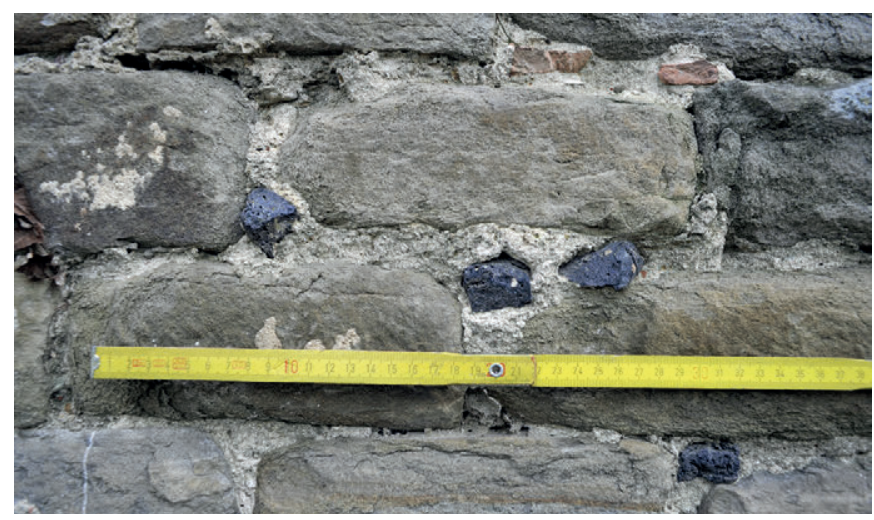

Fig. 5 e 6 . A sinistra, scacchiera composta da bozzette di Marmo Verde di Monte Calvi e Pietra Alberese presente sul prospetto nord della Pieve di Sant'Agata del Mugello; a destra, laterizi stracotti e vetrificati presenti come elemento decorativo nei giunti del campanile della Pieve di San Giovanni Maggiore.

decorativo. Infine si registra la presenza di calcari e di arenarie della Formazione di Sillano utilizzati come materiale costruttivo, sempre in concomitanza con la Pietra Alberese.

Dal confronto fra i dati crono-tipologici è possibile individuare quattro periodi storici legati a momenti costruttivi di particolare importanza. Ogni fase sembra caratterizzata da una scelta di uno o più materiali in riferimento a fattori di natura diversa, siano essi economico-politici o semplicemente pratici. In particolare potremmo concentrare l'utilizzo dei materiali da costruzione nei seguenti periodi costruttivi:

- I periodo (X-XII secolo): ad un motivo strettamente correlato alle caratteristiche geologiche del territorio sembra imputabile la scelta della materia prima per le costruzioni cronologicamente collocabili fra il X ed il XII secolo (fig.7). In questo periodo, nel quale possono essere inserite le edificazioni di quasi tutte le dodici strutture religiose interessate dalla ricerca, appare infatti chiara una netta demarcazione che vede l'impiego della Pietra Alberese nel Mugello Orientale e l'utilizzo della Pietra Serena e dell'Arenaria di Monte Senario nel Mugello Occidentale. Tale fenomeno sembra dunque correlabile allo sfruttamento puntuale delle risorse presenti nel sottosuolo dei due diversi contesti, piuttosto che alla valutazione da parte di maestranze e committenze delle caratteristiche (costruttive e decorative) delle singole materie prime. 


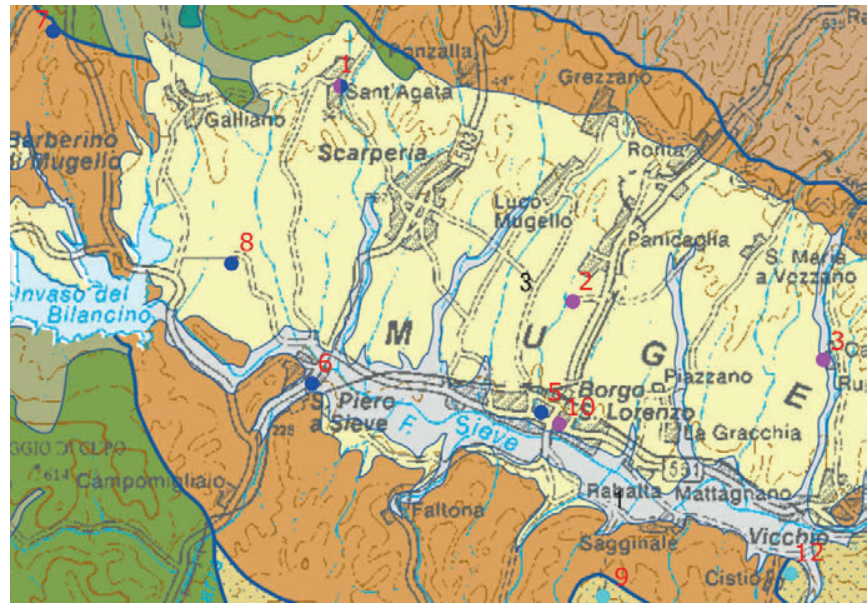

Fig. 7. Materiali da costruzione impiegati in Mugello dal X al XII secolo: Pietra Alberese (colore blu), Pietra Serena (colore magenta) e Arenaria di Monte Senario (colore ciano).

- II periodo (XIII-XIV secolo): in questo periodo ciò che appare estremamente chiaro dall'integrazione fra la lettura stratigrafica e l'analisi della Carta Geologica della Toscana è l'introduzione in una zona ben delineata del Mugello di un nuovo materiale costruttivo: il laterizio. E' infatti nella prima metà del XIII secolo che vengono attestate le più antiche costruzioni in mattoni ${ }^{4}$ ad opera di una committenza religiosa e, probabilmente, impiegando maestranze specializzate. L'analisi della struttura materiale denuncia infatti un periodo costruttivo collocato intorno alla metà del Duecento nel quale due chiese facenti parte di conventi francescani (San Francesco a Borgo e San Bonaventura del Bosco ai Frati) ed un altro edificio religioso, ubicato nei pressi degli altri due, che nel XIII secolo rivestiva una notevole importanza politico-amministrativa sul territorio (Pieve di San Lorenzo a Borgo), subiscono ristrutturazioni o intere ri-edificazioni in laterizio. L'ipotesi di una possibile interazione fra l'introduzione del nuovo materiale costruttivo in Mugello e la presenza di maestranze alloctone, viene alimentata dall'analisi stilistica ed archeologica del campanile della Pieve di Borgo San Lorenzo, la cui tipologia architettonica, come già ipotizzato da altri studiosi (Moretti-Stopani 1974:171, 190, 191), potrebbe far pensare ad influenze provenienti da contesti lombardi ${ }^{5}$ (fig. 11).

\footnotetext{
4 La datazione proposta deriva dalle analisi archeologiche operate sugli edifici del Mugello e dall'integrazioni di questi dati con quelli reperiti da numerose fonti storiche edite ed inedite (per maggiori informazioni si rimanda a Arrighetti 2015).

5 La possibile attestazione di elementi in stile lombardo nella Pieve di San Lorenzo a Borgo viene discussa nel successivo paragrafo relativo alle maestranze locali e specializzate operanti in Mugello.
}

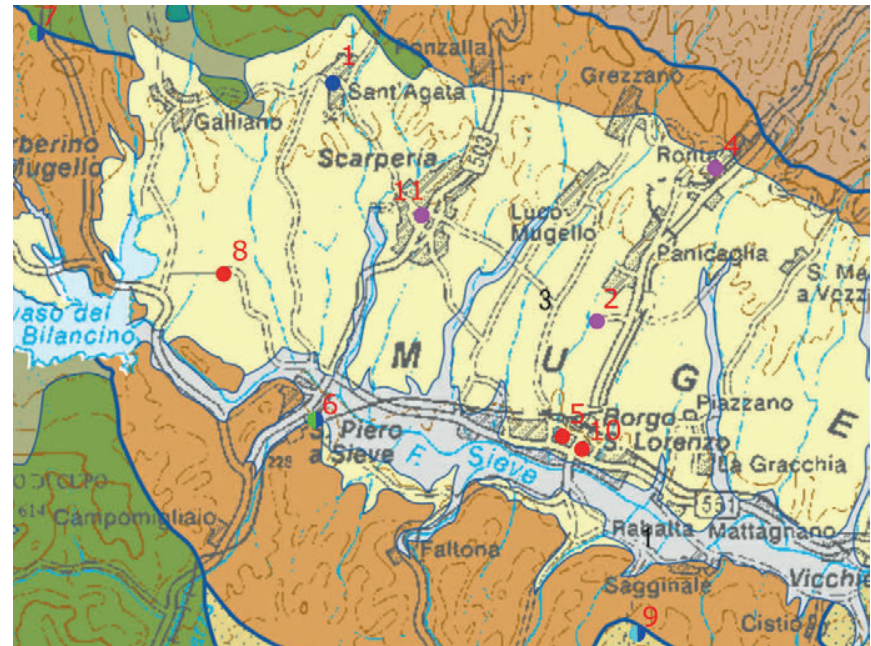

Fig. 8. Materiali da costruzione impiegati in Mugello nel XIII-XIV secolo: Pietra Alberese (colore blu), Pietra Serena (colore magenta), Laterizio (colore rosso) e Arenaria di Monte Senario (colore ciano).

Oltre al laterizio, in questo arco cronologico si possono riscontrare continuità nell'utilizzo della Pietra Serena e della Pietra Alberese nelle stesse zone analizzate nel precedente periodo costruttivo (fig.8). Il fenomeno è legato probabilmente ad un costante sfruttamento delle stesse aree di approvvigionamento delle materie prime, forse legate alla presenza delle medesime maestranze e committenze del periodo precedente.

- III periodo (XV secolo): questo periodo funge da ponte tra due grandi momenti costruttivi che interessano l'area del Mugello, ovvero gli estesi restauri operati nel Due-Trecento e le ricostruzioni post-sismiche operate dal 1542 alla prima metà del XVII secolo. Ciò che si nota è una continuità nell'utilizzo dei materiali costruttivi lapidei nelle zone descritte nel primo periodo, affiancate da un'introduzione del laterizio per alcuni restauri operati nel Palazzo dei Vicari di Scarperia ${ }^{6}$. In quest'ultimo caso potremmo forse ipotizzare un legame tra una committenza molto facoltosa, la famiglia de' Medici', e le maestranze specializzate coinvolte nelle costruzioni in laterizio del secondo periodo.

- IV periodo (XVI-inizi XVII secolo): nell'ultimo periodo sottoposto ad analisi vengono raccolti tutti i

\footnotetext{
L'edificio viene indicato in figura 9 con il numero 11.

In questo periodo sono numerose le attestazioni degli investimenti effettuato dalla famiglia de' Medici nel Mugello. Nel costituiscono validi esempi la Fortezza di San Martino a San Piero a Sieve, il patronato di numerose Pievi e chiese nel territorio, l'ampliamento dei poderi del Trebbio, di Cafaggiolo e di altre ville collocate nei comuni mugellani.
} 


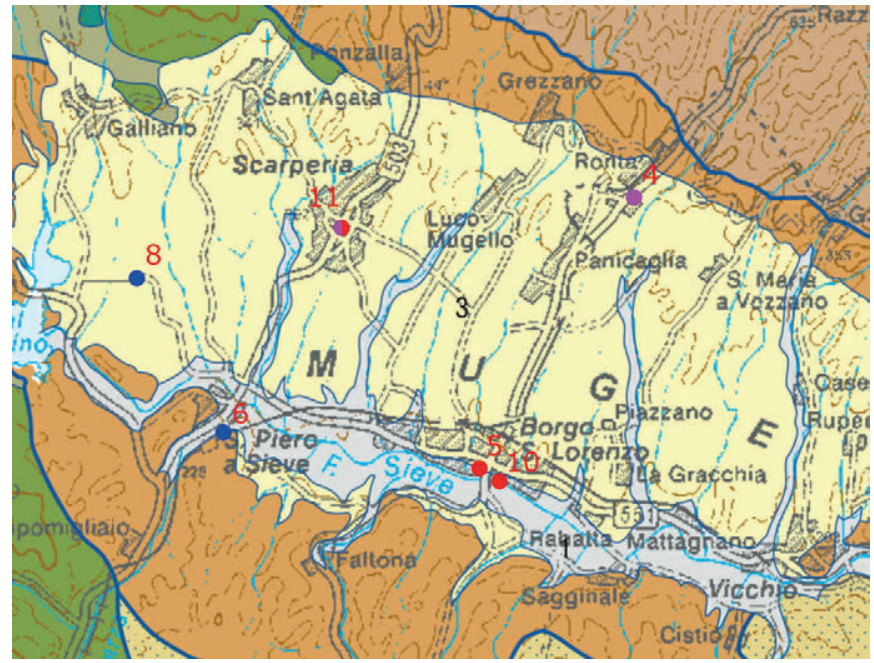

Fig. 9. Materiali da costruzione impiegati in Mugello nel XV secolo: Pietra Alberese (colore blu), Pietra Serena (colore magenta) e Laterizio (colore rosso)

restauri o le ricostruzioni operati a seguito degli eventi sismici del 1542, del 1597 e del 1611. In questo caso ciò che appare interessante è la totale assenza fra $i$ materiali costruttivi della Pietra Alberese, in contrapposizione ad un esteso utilizzo della Pietra Serena e del laterizio (fig.10). Il motivo della scelta preferenziale di queste due materie prime non appare chiaro. Possibili spiegazioni potrebbero derivare dall'abbandono delle zone di estrazione dell'Alberese in favore di un più esteso ed intensivo sfruttamento della Pietra Serena, materia prima utilizzata in modo preponderante dal Quattrocento in poi, sia come materiale costruttivo che per la produzione di elementi decorativi o architettonici, nell'edilizia rinascimentale e cinquecentesca fiorentina. Lo stesso vale per il laterizio che trova largo utilizzo come materiale costruttivo negli edifici in ambito fiorentino e nel contado, dal Cinquecento in poi.

In conclusione potremmo quindi definire alcuni aspetti essenziali legati alla società e ai modi di costruire che hanno caratterizzato l'edilizia storica del Mugello dai secoli centrali del Medioevo alla prima Età Moderna.

Per quanto riguarda i materiali lapidei sembra essere la disponibilità di una specifica materia prima ad influenzare in modo preponderante i materiali da costruzione utilizzati nel primo periodo di edificazione delle strutture religiose e nelle costruzioni o i restauri operati nei periodi seguenti. Le zone di estrazione, dove veniva operato uno sfruttamento intensivo di Pietra Arenaria e di Pietra Alberese (alla quale viene a coincidere un'estrazione ed utilizzo delle pietre della Formazione di Sillano), legate

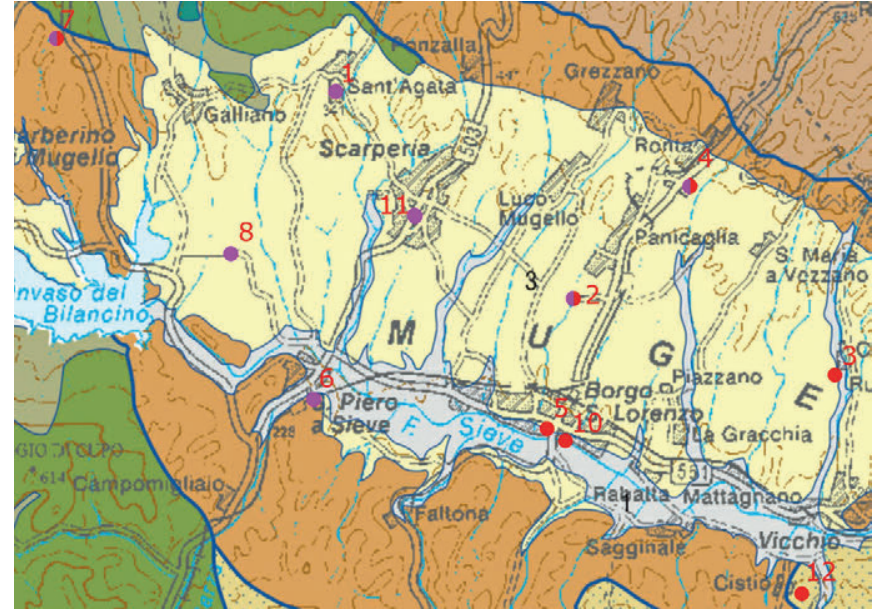

Fig. 10. Materiali da costruzione impiegati in Mugello nel XVI ed inizi XVII secolo: Pietra Serena (colore magenta) e Laterizio (colore rosso)

probabilmente alla disponibilità e funzionalità di queste materie prime, appaiono quindi i tratti caratterizzanti che influenzano l'architettura storica del Mugello. Purtroppo non essendo stata effettuata un' analisi sulle cave storiche presenti nell'area appare difficile ad oggi ipotizzare una relazione fra le differenti zone di approvvigionamento e la messa in opera delle materie prime.

Il laterizio rappresenta invece un elemento caratterizzante che permette di collegare l'utilizzo di una specifica materia prima con le esigenze di una committenza. L'ingaggio di maestranze specializzate, inoltre, si pone probabilmente alla base del sensibile cambiamento nella cultura costruttiva del Mugello non solo per l'introduzione di un nuovo materiale da costruzione nell'architettura, ma anche per le conseguenti innovazioni in campo tecnologico necessarie alla sua produzione.

\section{PRIME CONSIDERAZIONI PER UN "ATLANTE CRONO-TIPOLOGICO DELLE TECNICHE COSTRUTTIVE MURARIE TRADIZIONALI" DEL MUGELLO}

Confrontando i dati ottenuti dalla caratterizzazione delle murature individuate nell'analisi dei siti in Mugello, è stato possibile proporre alcune considerazioni in merito alle Tecniche Costruttive Murarie (TCM) utilizzate nei diversi periodi storici nel contesto in esame, in funzione della costituzione di un "Atlante Crono-Tipologico" delle stesse. Una prima fase del lavoro ha previsto una catalogazione delle diverse TCM, eseguita individuando, caratterizzando e datando in cronologia relativa le diverse murature mediante 
la lettura stratigrafica dei manufatti architettonici. A questa operazione è seguito il confronto fra i dati emersi dalle analisi archeologiche e le datazioni proposte dalle fonti storiche. Attraverso questo processo è stato possibile proporre una cronologia assoluta per le seguenti TCM:

- XI secolo:

- San Cassiano in Padule - TCM1

- San Gavino Adimari - TCM1

- San Giovanni Maggiore - TCM1

- San Pietro a San Piero a Sieve - TCM1

- XII secolo:

- Sant'Agata - TCM1

- Santa Maria a Fabbrica - TCM1

- Bosco ai Frati - TCM1

- Metà XIII secolo:

- San Francesco a Borgo - TCM1

- San Francesco a Borgo - TCM2

- San Gavino Adimari - TCM2

- Sant'Agata - TCM2

- San Pietro a San Piero a Sieve - TCM2

- 1420:

- Bosco ai Frati - TCM2

- 1443:

- San Francesco a Borgo - TCM3

- 1525-26:

- Sant'Agata - TCM4

- XVII secolo:

- San Francesco a Borgo - TCM4

- Post. 1777:

- San Pietro a San Piero a Sieve - TCM3

- Inizi XIX secolo:

- Sant'Agata - TCM3

- XX secolo:

- San Cassiano in Padule - TCM2

- San Gavino Adimari - TCM3

- Santa Maria a Fabbrica - TCM2

- Cronologia non disponibile:

- Sant'Agata - TCM5
Dalla catalogazione periodizzata delle Tecniche Costruttive Murarie del Mugello è possibile, confrontando le caratteristiche principali delle stesse ${ }^{8}$, proporre le seguenti riflessioni':

Apparecchiatura: L'apparecchiatura sembra essere uno dei parametri distintivi per comprendere i caratteri costruttivi adottati in Mugello nei diversi periodi storici. La posa in opera regolare viene attestata in otto casi, in un arco cronologico che spazia dall'XI agli inizi del XIX secolo. Le apparecchiature in corsi budinati, sia di bozze regolari in Pietra Alberese e in arenaria (Pietra Serena e Arenaria di Monte Senario) che di ciottoli di Pietra Serena, vengono attestate in quattro casi ed il loro utilizzo si concentra invece dall'XI al XIII secolo. L'apparecchiatura subisce un drastico decadimento nel XX secolo con pose in opera irregolari attestate nella maggior parte dei casi analizzati. Solo gli edifici monumentali localizzati nei centri cittadini (es. Pieve di Borgo San Lorenzo) sembrano discostarsi da questo fenomeno.

I giunti e letti di posa sembrano caratterizzarsi per range dimensionali piuttosto ampi nell'XI secolo (da 2.2 a $0.5 \mathrm{~cm}$ ) arrivando poi a dimensioni molto più contenute nel XII secolo (da 1.2 a 0.2 $\mathrm{cm}$ ) ed infine ad una sorta di standardizzazione per le costruzioni del 1420 e del 1525-26 (da 0.7 a 0.3 $\mathrm{cm})$. Dal XVIII secolo in poi, come per le apparecchiature, si assiste invece ad un trend negativo che porta ad un aumento delle misure, fino alla totale irregolarità dimensionale per il XX secolo. Mettendo in relazione il materiale costruttivo impiegato e le dimensioni dei giunti è possibile inoltre notare che la Pietra Alberese, materia prima per la quale abbiamo le maggiori attestazioni, quando utilizzata come unico materiale costruttivo si caratterizza per altezze dei giunti concentrate tra 1 a 0.3 centimetri, mentre quando ne viene documentato l'utilizzo in concomitanza della Pietra della Formazione di Sillano, presenta letti di posa con dimensioni fino a 2.4 centimetri.

\footnotetext{
8 I criteri adottati nella classificazione delle tecniche costruttive murarie fanno riferimento a Fiorani 1996a e Fiorani 1996b per la lavorazione dei materiali, Parenti 1987 e Mannoni 2005 per la classificazione delle apparecchiature murarie e per la loro caratterizzazione, Bessac 1986 per la finitura degli elementi lapidei 9 Dall'elenco è stata esclusa la materia prima poiché ampiamente trattata nel paragrafo precedente.
} 
Lavorazione: per quanto concerne la lavorazione dei materiali costruttivi, con particolare riferimento alla Pietra Alberese, materia prima maggiormente rappresentativa fra quelle proposte nei campioni analizzati, non vengono attestati decadimenti o miglioramenti nel lungo periodo. Viene infatti testimoniata una sostanziale continuità nell'utilizzo sia della squadratura che della sbozzatura. Le altezze e le larghezze risultano piuttosto irregolari, tranne nei casi dell'Arenaria di Monte Senario nella Chiesa di Santa Maria a Fabbrica e degli interventi in Pietra Alberese operati nel 1420 nella Chiesa del Convento del Bosco ai Frati, che invece si caratterizzano per misure estremamente standardizzate. L'utilizzo dei ciottoli di Pietra Serena non lavorati viene riportato con continuità dall'XI secolo fino ai restauri operati nel XX secolo. Dal Novecento in poi si nota infine un'assenza di lavorazione sui materiali messi in opera negli edifici analizzati.

Finitura: la finitura superficiale dei paramenti esterni viene ricostruita principalmente dall'analisi delle murature in Pietra Alberese, dove l'assenza di degrado superficiale ha permesso di ipotizzare, dalle tracce impresse sui conci, gli strumenti adottati in fase di finitura degli stessi. Gli strumenti più utilizzati sembrano essere quelli a lama piana, con un utilizzo continuativo dal XII al XIX secolo. Sporadica la presenza di spianatura esterna delle superfici, cronologicamente localizzata nel XII e nel XIII secolo per la sola Pieve di Sant'Agata, e l'utilizzo della subbia o altro strumento a punta, utilizzata in due casi nel XII secolo e nel 1420.

Mattoni: I dati sull'impiego dei laterizi in Mugello portano un'attendibilità piuttosto limitata. Le misure sono state infatti ricavate dall'analisi dei paramenti esterni ed interni di soli tre Complessi Architettonici su Fasi Costruttive datate in cronologia assoluta o relativa. In particolare i siti e le misure prese in considerazione per questa operazione sono i seguenti:

- Chiesa di San Francesco a Borgo San Lorenzo:

a) Paramento esterno - Fase 2 (metà XIII secolo): $28 / 29 \mathrm{~cm}$ x $12 \mathrm{~cm}$ x $5 \mathrm{~cm}$
- Chiesa di San Bonaventura del Bosco ai Frati:

a) Paramento esterno - Fase 2 (1245 ca.): 26 $\mathrm{cm} \times 16 / 16.5 \mathrm{~cm} \times 4.8 / 5.3 \mathrm{~cm}$

- Pieve di San Lorenzo a Borgo San Lorenzo:

a) Interno della Pieve - Archi della navata destra (cronologia non definibile): $45 / 48$ $\mathrm{cm} \times 23.5 \mathrm{~cm} \times 5.3 / 5.8 \mathrm{~cm}$

b) Interno del campanile - Fase 2 (125863): $28 / 29 \mathrm{~cm} \times 12 \mathrm{~cm} \times 5.5 / 6 \mathrm{~cm}$

c) Interno del campanile - Fase 2 (125863): $28 / 29 \mathrm{~cm} \times 12 \mathrm{~cm} \mathrm{x} 4.5 / 5.2 \mathrm{~cm}$

d) Interno del campanile - Fase 4 (post. 1263 - ant. 1542): $25 / 26.5 \mathrm{~cm} \mathrm{x} 10.5 / 11 \mathrm{~cm} \mathrm{x}$ $5 / 5.5 \mathrm{~cm}$

e) Interno del campanile - Fase 6 (post. 1263 - ant. 1542): $25.5 \mathrm{~cm} \times 11 / 11.5 \times 5 / 6.5 \mathrm{~cm}$

f) Interno del campanile - Fase 8 (1542): $26.5 / 27 \mathrm{~cm} \times 11.5 / 12.5 \mathrm{~cm} \times 5.2 / 6 \mathrm{~cm}$

Come è possibile ricostruire dalle misure appena descritte, i dati estremamente eterogenei ricavati dall'analisi dei paramenti dei siti analizzati, fanno presumere per il Mugello la presenza di metodi di produzione di mattoni non standardizzati. L'ampio range che sussiste fra le grandezze minime e massime dei parametri sottoposti ad indagine permette quindi di escludere la possibilità di ottenere tipologie di riferimento dalle tecniche murarie analizzate. Per questo motivo, i dati ricavati dai paramenti in mattoni non sono stati utilizzati nell'interpretazione dell'evoluzione dei sistemi costruttivi del contesto di studio. Le informazioni elaborate hanno costituito invece un supporto all'interpretazione e alla datazione delle tecniche costruttive murarie in materiali litici, costituite da basi di dati molto più solide.

Mettendo in relazione le caratteristiche che compongono le diverse tecniche analizzate, è possibile proporre un "Atlante Crono - Tipologico delle Tecniche Costruttive Murarie del Mugello", uno strumento in grado di mettere in luce i processi di lavorazione e messa in opera utilizzati dalle maestranze nei diversi periodi storici. Questo ci permette a sua volta di arrivare ad ipotesi inerenti i caratteri costruttivi degli edifici presenti in Mugello e i fattori economico-politici o di altra natura alla base delle scelte operate dalla committenza. 


\section{TIPO A (XI secolo - 1525-26)}

Muratura in Pietra Alberese, sporadicamente alternata ad altri litotipi come la Pietra Serena e la Formazione di Sillano, messa in opera attraverso un'apparecchiatura regolare formata da conci squadrati di altezza compresa fra 20 e $26 \mathrm{~cm}$ e larghezze eterogenee, con giunti compresi tra 0.2 e $1.4 \mathrm{~cm}$ e con finitura esterna operata con strumento a lama piana e sporadicamente con subbia. Ne fanno parte: Sant'Agata del Mugello - TCM1; Bosco ai Frati - TCM2; Sant'Agata del Mugello - TCM4.

\section{TIPO B (XI - XIII secolo)}

Muratura in Pietra Alberese, talvolta alternata a litotipi della Formazione di Sillano, messa in opera con apparecchiatura regolare, formata da pietre sbozzate o squadrate, di altezza compresa fra 12 e $21 \mathrm{~cm}$ e larghezza tra 36 e $45 \mathrm{~cm}$, con giunti di dimensioni che spaziano da 0.5 a $2.4 \mathrm{~cm}$ e con finitura esterna operata con strumento a lama piana. $\mathrm{Ne}$ fanno parte: San Pietro a San Piero a Sieve - TCM1 e TCM2; Bosco ai Frati - TCM1; Sant'Agata - TCM2.

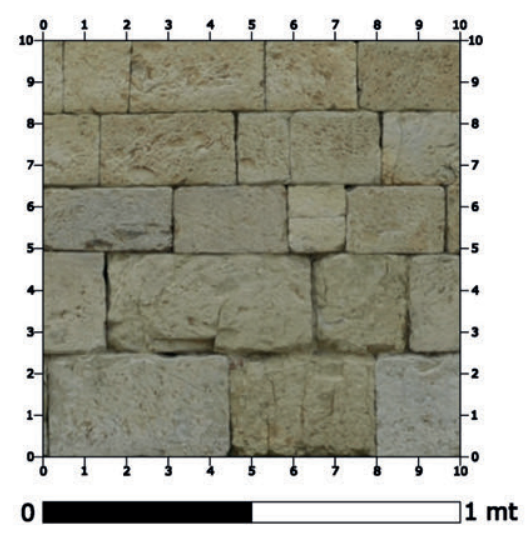

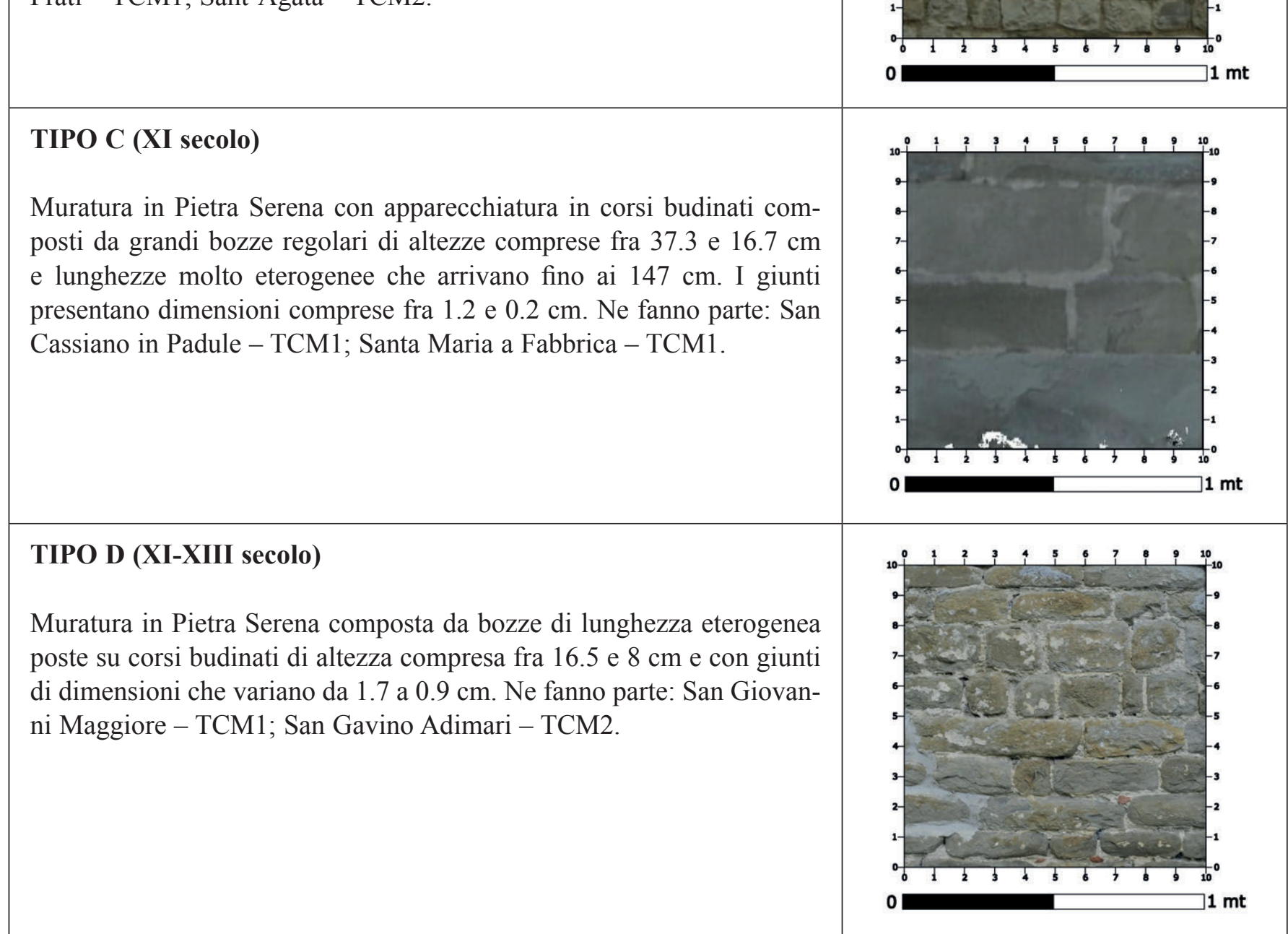




\section{TIPO E (XII secolo)}

Muratura in Arenaria di Monte Senario composta da conci squadrati, di altezza compresa fra 37 e $32 \mathrm{~cm}$ e lunghezza tra 70 e $38 \mathrm{~cm}$, posti in opera attraverso un'apparecchiatura regolare con giunti e letti di posa molto precisi (gli spessori variano da 0.7 a $0.1 \mathrm{~cm}$ ). Ne fa parte: San Gavino Adimari - TCM1.

\section{TIPO F (Post. 1777)}

Muratura a ricorsi orizzontali e paralleli sdoppiati di Pietra Alberese e Laterizi. I litotipi si presentano sbozzati e talvolta spaccati, con finitura esterna operata attraverso strumento a lama piana e con altezze comprese tra 18 e $11 \mathrm{~cm}$ e larghezze tra 43 e $9.6 \mathrm{~cm}$. Ne fa parte: San Pietro a San Piero a Sieve - TCM3.

\section{TIPO G (XX secolo)}

Muratura irregolare composta principalmente da ciottoli e materiale da costruzione di reimpiego (sia laterizi che elementi litici in arenaria). $\mathrm{Ne}$ fanno parte: San Cassiano in Padule - TCM2; San Gavino Adimari TCM3; Santa Maria a Fabbrica - TCM2.
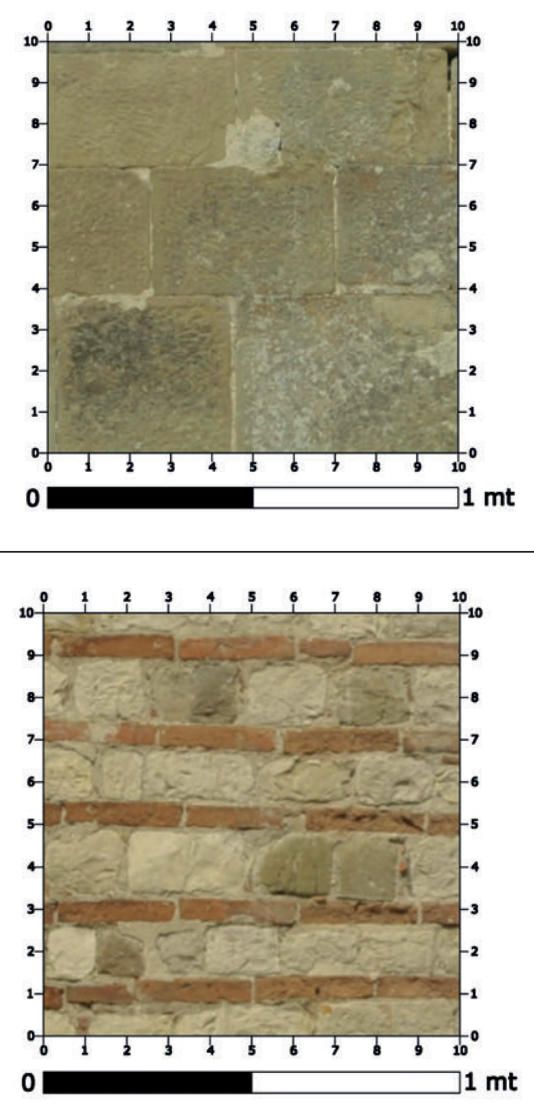

\section{IL RUOLO DELLE MAESTRANZE LOCALI E SPECIALIZZATE NELLA COSTRUZIONE DEGLI EDIFICI}

Per ricostruire il ruolo dei soggetti attivi nelle costruzioni e negli interventi operati sugli edifici del Mugello nei diversi periodi storici è di fondamentale importanza integrare i dati ottenuti dalle analisi effettuate sulle tecniche costruttive murarie e sull'approvvigionamento e l'utilizzo dei materiali da costruzione, con i risultati emersi dalle analisi storico-archeologiche effettuate sui contesti storici di riferimento.
I risultati vengono suddivisi nei periodi cronologici che interessano i principali momenti costruttivi della zona:

XI-XII secolo: i dati ricavati dall'analisi dell'approvvigionamento ed utilizzo dei principali materiali costruttivi, questi ultimi derivati per lo più da materie prime cavate in loco, non sembra un criterio utilizzabile nella comprensione delle scelte operate dalle maestranze e dalle committenze per l'erezione degli edifici in questo arco cronologico. Piuttosto appare un elemento distintivo l'impiego, seppur sporadico, di materiali specifici reperibili più o meno nelle vicinanze dal luogo di 
costruzione. E' questo l'esempio del Marmo Verde di Monte Calvi per la Pieve di Sant'Agata, elemento che denota una volontà ben precisa da parte dei committenti nella scelta del materiale costruttivo da utilizzare. Oppure il laterizio stracotto e vetrificato impiegato come materiale decorativo nei giunti del campanile della Pieve di San Giovanni Maggiore, altro elemento che rappresenta un unicum fra i materiali utilizzati negli edifici del Mugello.

I parametri desunti dall'analisi delle tecniche costruttive murarie sembrano essere solo in parte di aiuto per la definizione dei ruoli dei soggetti coinvolti nelle costruzioni operate in questo periodo storico. L'analisi delle murature, ad esempio, testimonia una preminenza della squadratura e sbozzatura di materiali costruttivi di medio - grandi dimensioni per l'apparecchiatura di paramenti regolari, indice probabilmente della presenza di maestranze specializzate, forse di estrazione locale. La finitura superficiale delle pietre invece si lega principalmente alle caratteristiche della materia prima (se arenaria o calcarenite) piuttosto che alla tipologia di maestranza o committenza.

XIII secolo: il fattore preponderante che caratterizza questo periodo costruttivo sembra essere l'utilizzo del laterizio. Dal punto di vista tipologico, le misure estremamente eterogenee delle murature in mattoni non permettono la proposizione di specifiche categorie. L'impiego del mattone negli edifici storici trova però attestazioni storiche e stratigrafiche ben precise, che portano a definire una sequenza storica nel suo utilizzo. In particolare dopo una prima attestazione di questo materiale intorno alla metà del XIII secolo, utilizzato per la ri-edificazione del Convento di San Francesco a Borgo, il mattone vene impiegato in un periodo immediatamente successivo, probabilmente ascrivibile alla seconda metà del Duecento, negli ingenti restauri operati nei due conventi francescani presenti in Mugello (San Francesco a Borgo e Bosco ai Frati) e nella ricostruzione del campanile della Pieve di Borgo San Lorenzo. Da quel momento il mattone entra a far parte dei materiali da costruzione dell'area, arrivando nella seconda metà del XVI secolo a sostituire la Pietra Alberese. L'introduzione del laterizio, dunque, potrebbe essere stata operata nel corso del XIII secolo in relazione alla costruzione del convento di San Francesco a Borgo San Lorenzo, edificato da maestranze delle quali non abbiamo testimonianze storiche, probabilmente in concomitanza con la venuta del santo stesso in Mugello (Altieri 2009: 127).
L'unica considerazione sui costruttori impiegati nel corso del Duecento per la produzione e messa in opera dei laterizi, deriva dal confronto tipologico fra il campanile della Pieve di Borgo San Lorenzo (fig.11), costruito tra il 1258 e il 1263 , e i campanili in stile lombardo presenti nel Centro e nel Nord Italia. La similitudine morfologica fra queste costruzioni potrebbe costituire la testimonianza della circolazione di maestranze lombarde in Mugello nel XIII secolo. Un fenomeno che avrebbe portato alla conseguente introduzione del laterizio fra i materiali costruttivi impiegati negli edifici dell'area nel Duecento, fenomeno già ampiamente documentato per altre zone della Toscana da numerosi lavori editi, incentrati sia sullo studio delle fonti scritte (Salvestrini 2011: 37) che sull'analisi delle evidenze materiali (Bianchi 1996; Frati 2013a, 2013b; Parenti e Quiros Castillo 2000; Pittaluga e Quiros Castillo 1997; Quiros Castillo 1997, 2001). Per questo motivo appare possibile che l'introduzione di questo nuovo materiale da costruzione possa essere messo in rapporto all'arrivo di costruttori specializzati dal Nord, per poi, vista la rapida espansione nel suo utilizzo, entrare a far parte de know-how delle maestranze locali nel corso del XIV e XV secolo.

Per quanto riguarda la pietra è invece possibile proporre riflessioni precise dalle tecniche costruttive analizzate. L'esempio che forse meglio testimonia l'impiego di maestranze specializzate in questo periodo è la Pieve di Sant'Agata del Mugello dove il materiale costruttivo impiegato, ovvero la Pietra Alberese, viene messo in opera attraverso una squadratura molto ben eseguita e una spianatura superficiale dei conci; tali fattori potrebbero quindi testimoniare una notevole capacità nella lavorazione dei materiali da parte di costruttori specializzati, probabilmente ingaggiati da parte di committenze con una certa disponibilità economica. In altri casi, come ad esempio nella Pieve di San Gavino Adimari, le operazioni di restauro furono messe in opera attraverso l'impiego di materie prime locali, sbozzate o spaccate e con apparecchiature tendenti alla regolarità. Al momento nessuna fonte ci testimonia l'impiego di maestranze specializzate o locali e la committenza che commissionò questi lavori.

Seconda metà XVI-inizi XVII secolo: questo periodo è riferibile alle intense ricostruzioni avvenute in seguito ai terremoti del 1542, del 1597 e del 1611. In questo caso i modi di intervenire sugli edifici, testimoniati direttamente dai metodi costruttivi impiegati, ci riportano un panorama eccezionalmente chiaro delle 


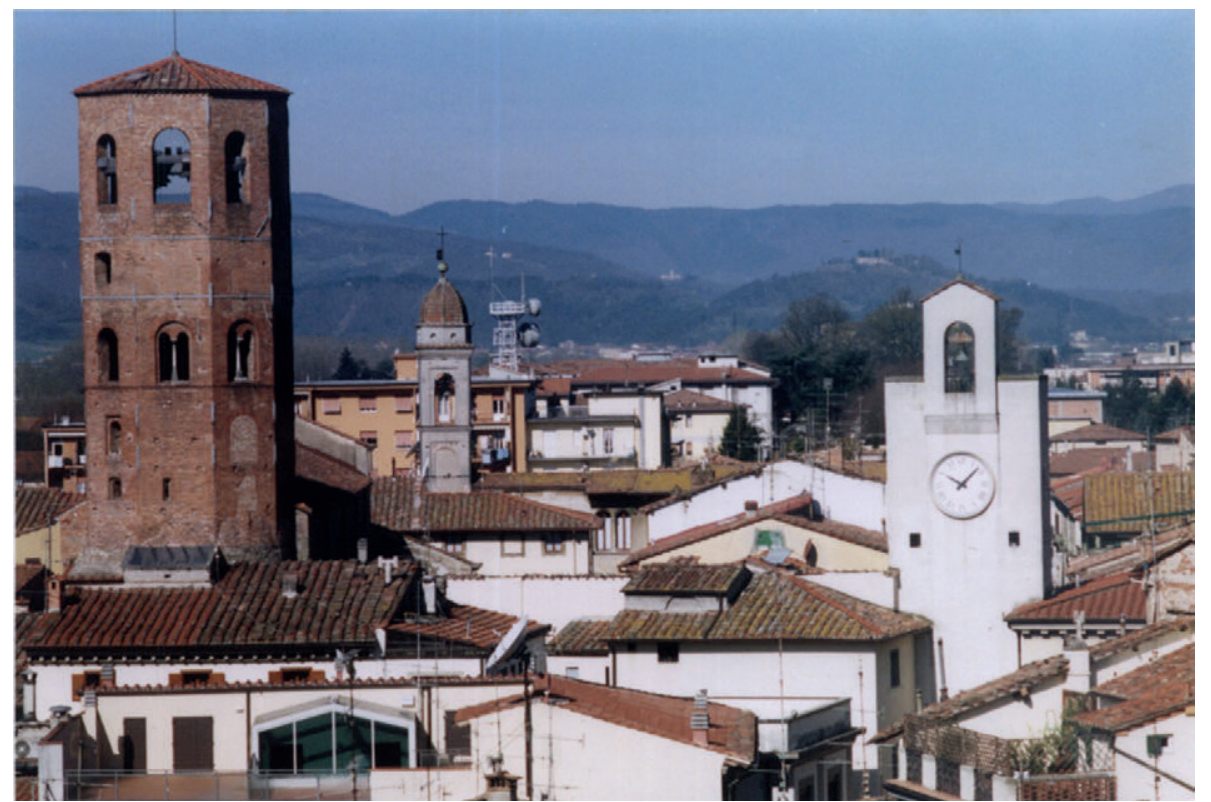

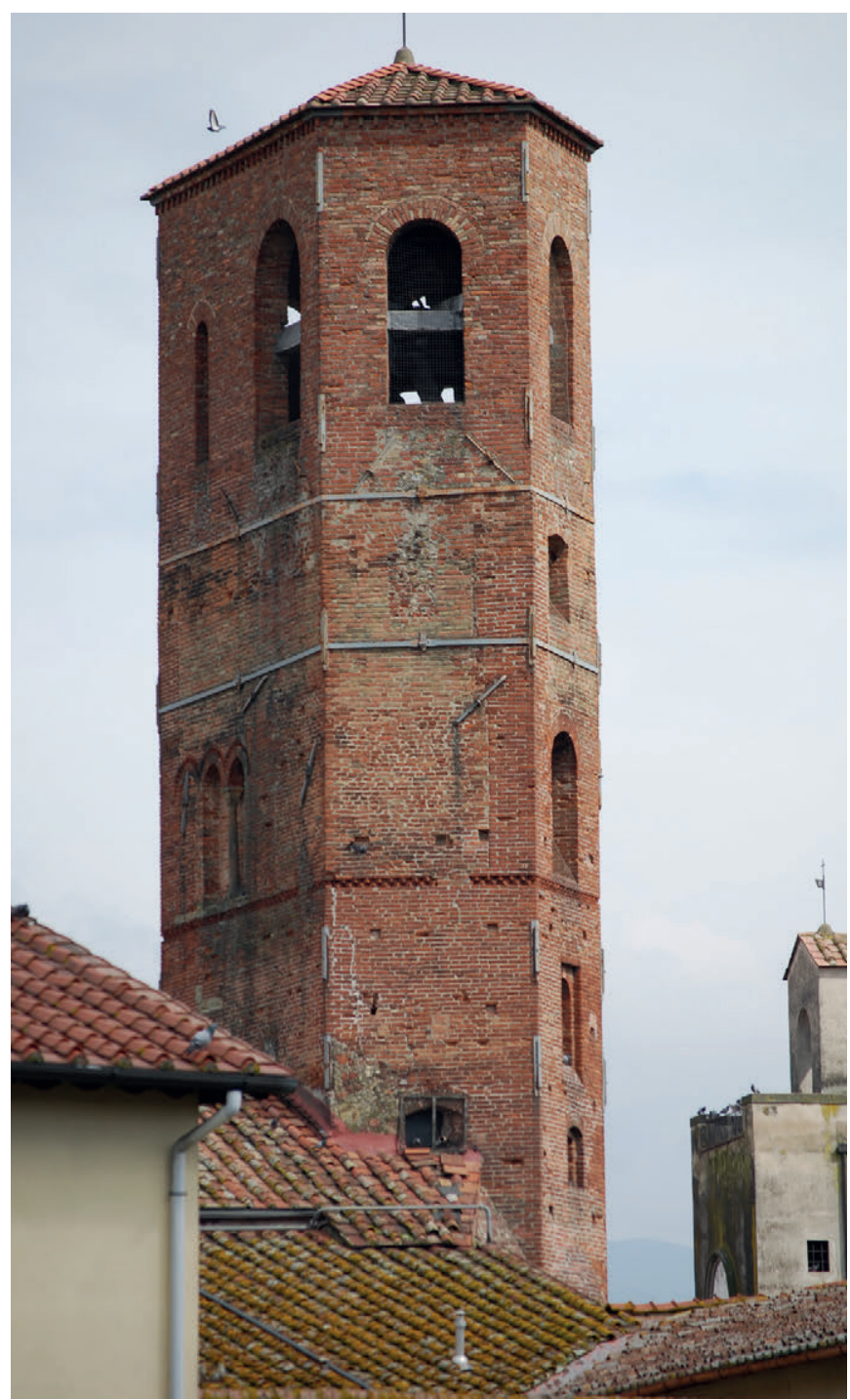

Fig. 11. Il campanile della Pieve di Borgo San Lorenzo. maestranze e delle committenze coinvolte. Per la maggior parte degli edifici attestati, ai meccanismi di danno attivati dai sismi non fecero seguito momenti costruttivi di una certa importanza. Al contrario, si attestano molto frequentemente ricostruzioni mirate a risolvere singoli 'problemi' (ad esempio la risarcitura delle lesioni, l'inserimento di catene, etc.), effettuate con materiali di spoglio o con materie prime miste, messe in opera mediante apparecchiature piuttosto irregolari. In un solo caso le fonti scritte ${ }^{10}$ ci testimoniano l'intervento di una committenza specifica, rappresentata dalla famiglia de' Medici, che utilizzò maestranze specializzate locali per gli interventi operati sulla Pieve di San Gavino Adimari. In questo caso infatti le ricostruzioni operate sulla chiesa e sul campanile mettono in evidenza una volontà forte da parte della committenza di donare un nuovo splendore all'edificio posto sotto il proprio patronato (fig.12).

La Pieve di Sant'Agata costituisce un contesto dove le intense ricostruzioni operate a cavallo tra il XVI ed il XVII secolo, testimoniate sia attraverso le fonti scritte ${ }^{11}$ che dalla lettura stratigrafica dell'edificio ${ }^{12}$, vengono

\footnotetext{
10 Per quanto riguarda le fonti scritte che, seppur posteriori, testimoniano l'intervento della famiglia de' Medici nel restauro di San Gavino: Baccini 1893; Baldi 1878. La ristrutturazione dell'edificio operata dalla famiglia Medici è testimoniata inoltre dalla presenza dello stemma della Famiglia in numerosi punti della chiesa.

11 Le testimonianze più importanti relative ai lavori di restauro operati sulla chiesa di Sant'Agata nel XVI secolo provengono da documenti scritti dall'allora Pievano Nozzolini e conservati presso l'archivio parrocchiale. I documenti sono stati recentemente pubblicati in un volume sulla storia di Sant'Agata (Brunori Cianti 2011)

12 Per maggiori dettagli sulla lettura stratigrafica della Pieve di Sant'Agata: Arrighetti 2015.
} 


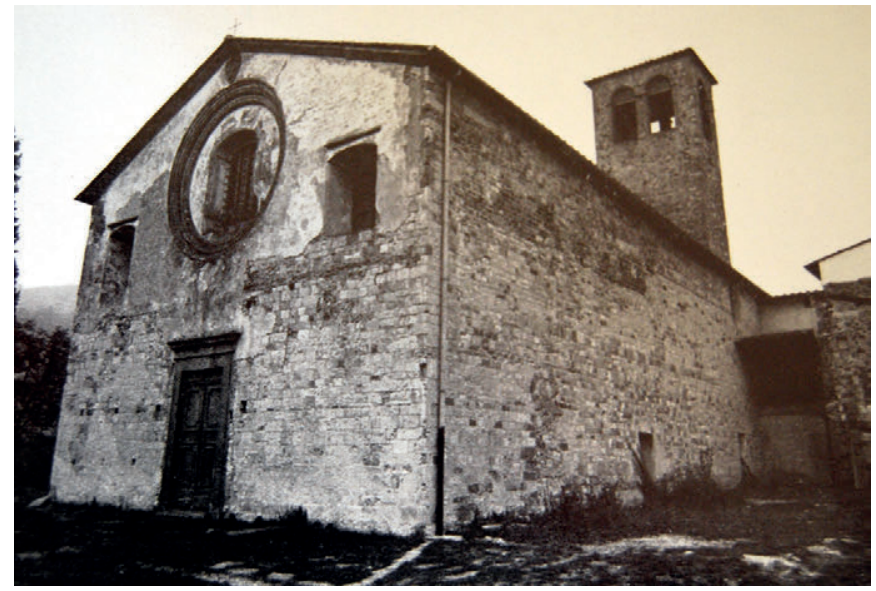

Fig. 12. La Pieve di San Gavino Adimari mostra ancora oggi caratteri cinquecenteschi e settecenteschi relativi ai due grandi restauri che hanno interessato la struttura in epoca moderna. (Campidori 1980: 100)

operate da due diverse committenze e maestranze. Il primo intervento operato sulla chiesa a seguito del sisma del 1542 fu eseguito dagli abitanti del centro demico di Sant'Agata, che risarcirono ed in parte ricostruirono, con materiali eterogenei e di spoglio, il campanile della chiesa $^{13}$ (fig.13). Un secondo momento costruttivo fu invece commissionato dal Pievano Nozzolini che ingaggiò maestranze locali e specializzate per operare ad

13 Il Pievano Nozzolini descrive in questo modo i lavori effettuati sul campanile della Pieve di Sant'Agata a seguito del sisma del 1542: “...Il campanile di questa Pieve ... l'anno 1542 per il tremuoto rovinò, e gli uomini della Compagnia della Visitazione ricopersono a uso di capanna con un poco di tettuccio quella parte che restò in piedi tutta screpolata, e quella facciata che guarda verso la chiesa la alzarono su sopra il tetto e vi fecero le finestre delle campane e lo ridessero alla forma che di contro si vede..." (Brunori Cianti 2011: 88).

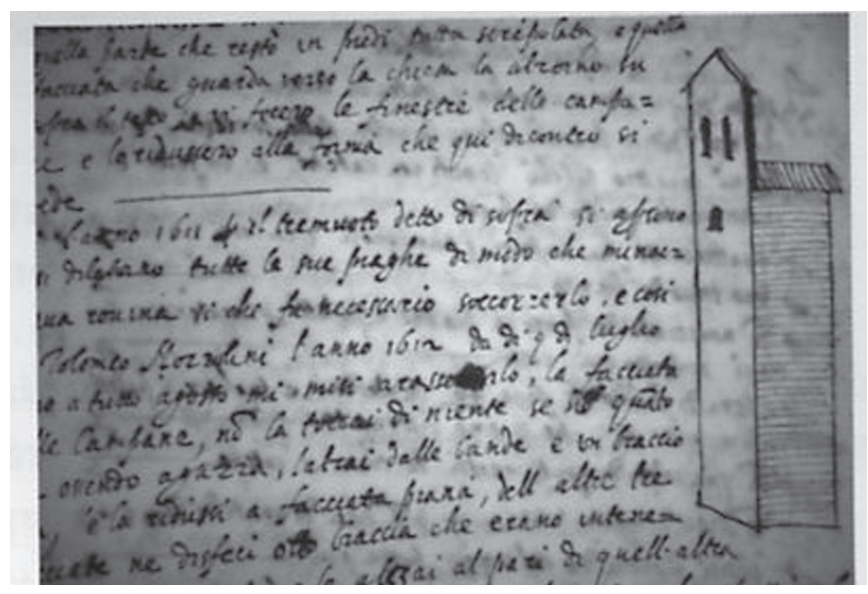

una vera e propria riedificazione di parte della chiesa e del campanile, utilizzando come materiali costruttivi la Pietra Alberese e la Pietra Serena (fig.14). In questo caso dunque gli interventi eseguiti a seguito dei sismi avvenuti nel 1542 e nel periodo 1597-1611 si differenziano notevolmente tra loro sia per le materie prime utilizzate che, in particolar modo, per la lavorazione e finitura dei materiali costruttivi messi in opera.

In linea generale furono quindi le disponibilità economiche delle diverse committenze e, di conseguenza, le maestranze locali più o meno specializzate ingaggiate nei lavori post-terremoto, che influenzarono in modo preponderante le ricostruzioni eseguite in questo arco cronologico.

XX secolo: l'evento principale che in questo periodo portò ad intense modifiche nella struttura materiale degli edifici fu il terremoto del 1919. A seguito dell'evento furono messi in opera numerosi interventi che spaziarono dalla risarcitura di quadri fessurativi più o meno importanti, fino alla completa ricostruzione di alcuni edifici danneggianti dall'evento tellurico. In particolare viene attestato, sia dalle fonti scritte che dalle analisi archeologiche, l'evidente volontà da parte dell'autorità centrale di riportare allo splendore determinate strutture, operando vere e proprie riedificazioni attraverso trasformazioni interne ed esterne degli edifici. E' il caso ad esempio della Pieve di Borgo San Lorenzo che fu quasi totalmente ricostruita o delle Pievi di San Cassiano in Padule e di San Cresci in Valcava che invece furono demolite per essere ricostruite dalle fondamenta (figg. 15, 16 e 17).

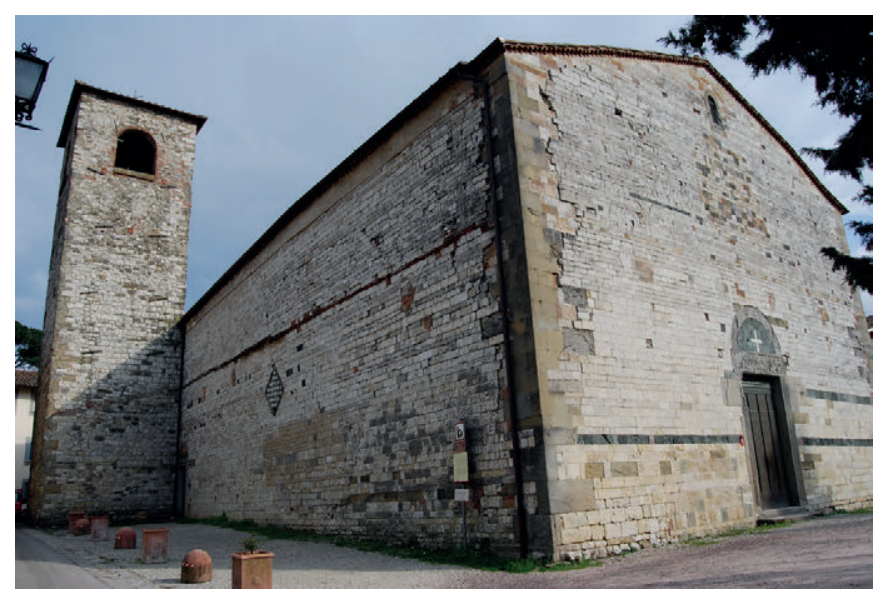

Fig. 13 e 14. A sinistra, il disegno della morfologia del campanile della Pieve di Sant'Agata del Mugello a seguito dei restauri operati dopo il sisma del 1542 ad opera di maestranze locali (Brunori Cianti 2011: 88). A destra, la Pieve di Sant'Agata in una foto del 2009. 


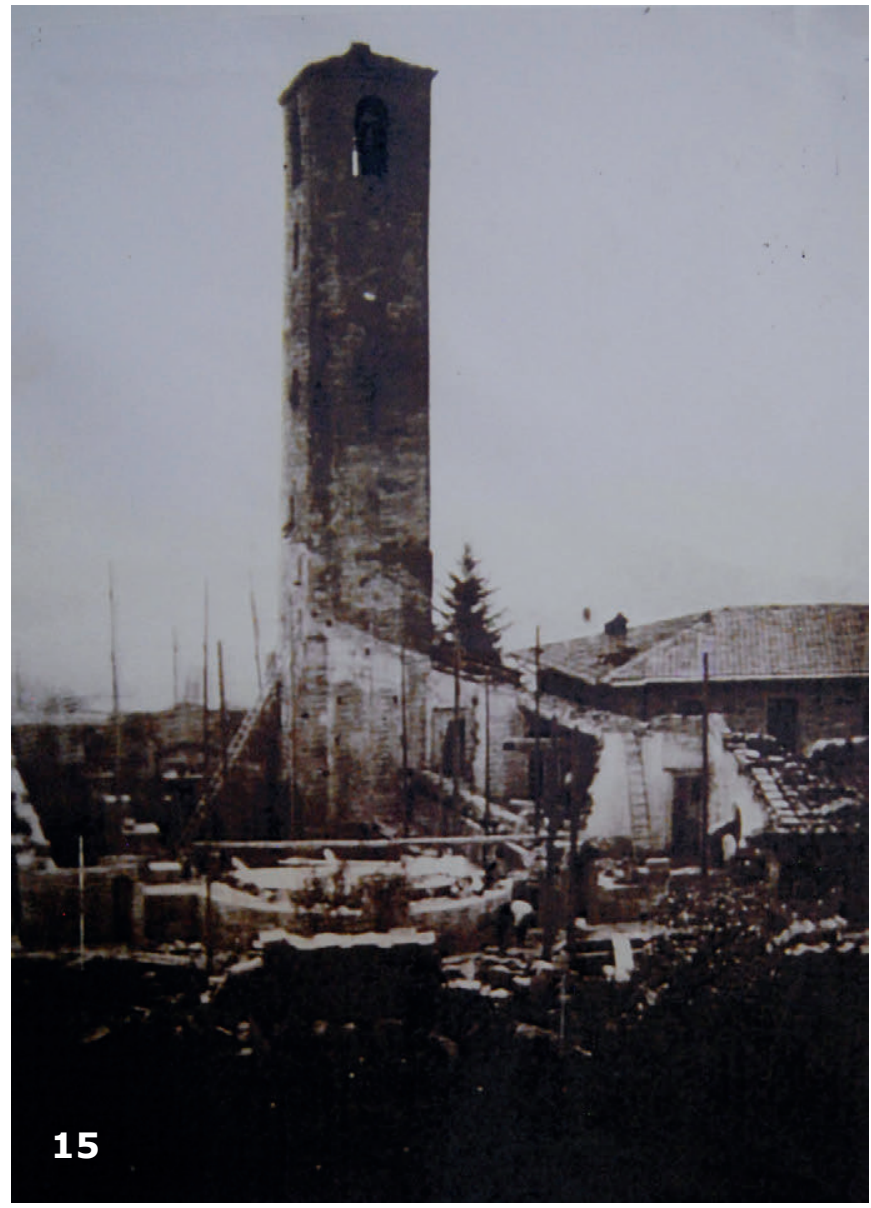

Fig. 15, 16 e 17. La riedificazione della Pieve di San Cresci in Valcava mostrata attraverso tre foto: a sinistra, a seguito del sisma del 1919 (Castenetto e Sebastiano 2004); nella foto centrale, durante i lavori di ricostruzione degli anni '20 (Castenetto e Sebastiano 2004); a destra, in un'immagine del 2009.

Discorso a parte invece meritano alcune chiese che, probabilmente a causa della loro posizione topografica isolata, della mancanza di fondi o del loro stato di abbandono, furono solo parzialmente restaurate in attesa di interventi successivi. Fra queste meritano di essere citate la Pieve di San Gavino Adimari, la Chiesa di Santa Maria a Fabbrica e la Chiesa di San Bonaventura del Bosco ai Frati.

\section{CONCLUSIONI}

Committenze, maestranze e materie prime sono i tre elementi che si pongono alla base delle principali scelte operate in Mugello nei diversi periodi storici per edificazioni ed interventi di vario tipo (dai restauri alle
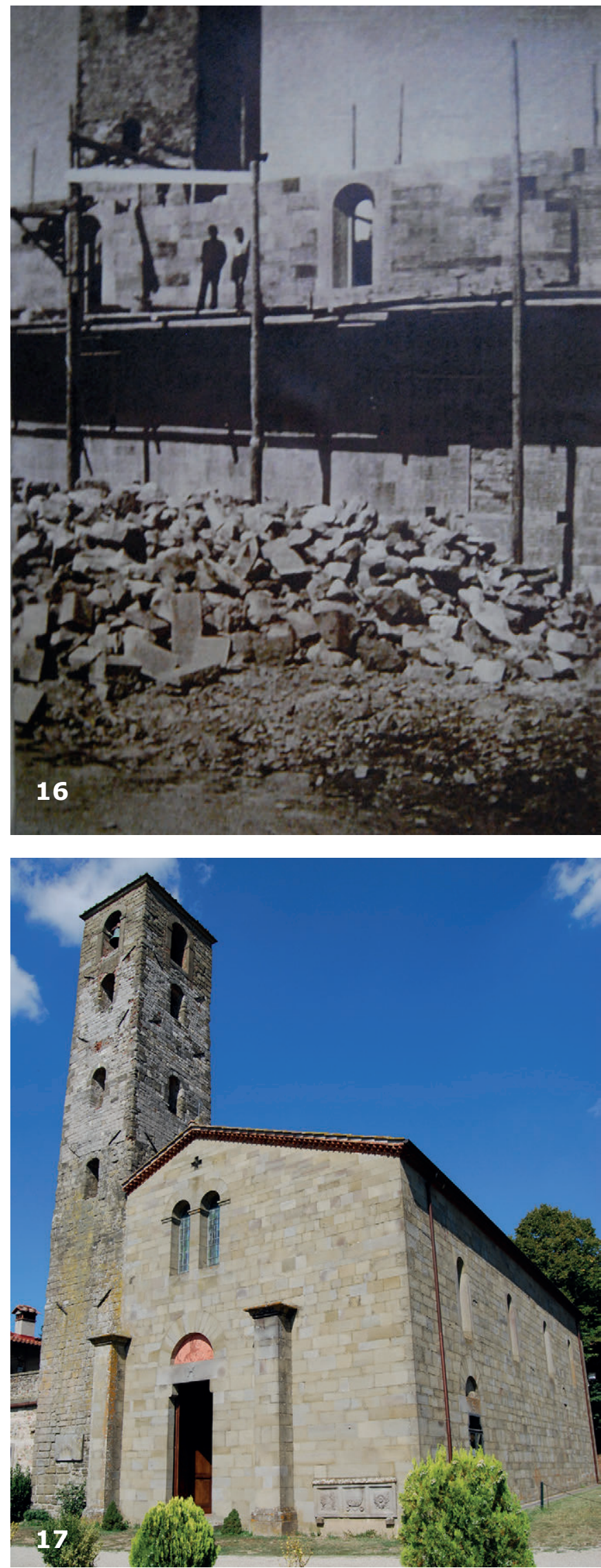
ricostruzioni). Dal confronto fra i risultati emersi nella ricerca, sembrano essere proprio questi fattori ad offrire la possibilità di comprendere come e perché si sia costruito in un luogo in un determinato periodo storico.

Le committenze, per lo più di estrazione religiosa, hanno spesso fatto ricorso a maestranze di tipo specialistico, probabilmente già presenti ed operanti sul territorio, in tutti i periodi storici analizzati. Di contro l'utilizzo di maestranze alloctone sembra probabilmente documentato intorno alla metà del XIII secolo per la sola costruzione del campanile della Pieve di Borgo San Lorenzo, dove il modello edilizio riporta caratteristiche architettoniche correlabili allo stile lombardo.

L'utilizzo delle materie prime sembra legato a fattori prettamente tecnico-pratici. La disponibilità e lo sfruttamento di affioramenti di specifici materiali costruttivi e l'introduzione di conoscenze costruttive economicamente e tecnicamente più vantaggiose di quelle in uso (pensiamo all'introduzione del laterizio che nel corso di tre secoli sostituisce la Pietra Alberese) si pongono come basi nelle scelte operate da committenze e maestranze nei periodi analizzati.

Un discorso a parte meritano le situazioni post-sismiche dove sembra emergere una sorta di differenziazione fra gli interventi eseguiti nei diversi periodi e contesti analizzati. Nel XVI secolo gli edifici testimoniano una duplice realtà: ai restauri frettolosi effettuati qualche giorno dopo un dato evento tellurico, messi in opera dagli stessi abitanti per risolvere $i$ danni più evidenti causati dal terremoto (es. la risarcitura della lesioni, la parziale ricostruzione di tetti, etc.) con tecniche costruttive piuttosto approssimative, fanno seguito interventi più specializzati eseguiti anche ad un secolo di distanza dagli eventi e legati alle richieste di specifiche committenze (es. la famiglia de' Medici, alcuni pievani, etc.). In questo secondo caso l'accuratezza nella scelta, nella lavorazione e nell'apparecchiatura dei materiali costruttivi denota la presenza di maestranze specializzate, probabilmente di estrazione locale. Nel XX secolo la situazione cambia nuovamente. A seguito del terremoto del 1919 sono gli edifici di maggior pregio e localizzati in centri cittadini a subire i restauri di maggiore entità. I complessi architettonici isolati vengono invece abbandonati o semplicemente messi in sicurezza attraverso provvedimenti temporanei che divengono invece definitivi, portando in breve tempo le strutture a situazioni di criticità strutturale.

\section{BIBLIOGRAFIA}

Altieri, A. 2009: Notizie del Borgo San Lorenzo in Mugello e suo territorio raccolte da Valentino Felice Mannucci nel 1742-1743, Colle Val d'Elsa.

Arrighetti, A. 2015: L'archeosismologia in architettura. Per un manuale, Firenze

Baccini, G. 1893: La pieve di San Gavino Adimari presso Barberino di Mugello. Memoria del P. Pompeo Franchi, in "Giotto. Bollettino Storico Artistico Letterario del Mugello", Firenze.

Baldi, F. 1878: La pieve di San Gavino Adimari e Benedetto Varchi : ricordi storici, Firenze.

Bessac, J.C. 1986: L'outillage traditionel du tailler de pierre de l'antiquité à nos jours, Parigi.

Bianchi, G. 1996: Maestri costruttori lombardi nei cantieri della Toscana centro-meridionale (secoli XII-XV). Indizi documentari ed evidenze materiali, in S. Della Torre, T. Mannoni e V. Pracchi, "Magistri d'Europa: eventi, relazioni, strutture della migrazione di artisti e costruttori dai laghi lombardi", Atti del Convegno, Como 23-26 ottobre 1996, Como, pp.155-166.

Brunori Cianti, L. 2011: Sant'Agata: una santa, una pieve, una comunità del Mugello, Firenze.

Campidori, P. 1980: Le chiese del Mugello, Firenze.

Castenetto, S. e Sebastiano, M. (a cura di) 2004: Mugello 29 giugno 1919. Sui luoghi del terremoto, pubblicazione a cura della Regione Toscana e della Protezione Civile, Firenze.

Fiorani, D. 1996a: Tecniche costruttive murarie medievali: il Lazio meridionale, Roma.

Fiorani, D. 1996b: Le tecniche costruttive murarie medievali del Basso Lazio. Metodo e percorsi di una ricerca, in S. Della Torre (a cura di), "Storia delle tecniche murarie e tutela del costruito. Esperienze e questioni di metodo", Milano, pp. 97-112.

Frati, M. 2013a: Verso un atlante delle murature ad Empoli: la mensiocronologia del laterizio, in "Milliarium", vol.10, Empoli, pp.126-135.

Frati, M. 2013b: Tracce lombarde nella Toscana protoromanica, in "Architettura dell'XI secolo nell'Italia del Nord. Storiografia e nuove ricerche", Atti del Convegno Internazionale di Studi, Pavia, 8-10 aprile 2010, Pisa, pp. 253-270.

Mannoni, T. 2005: "Archeologia della produzione architettonica. Le tecniche costruttive", Arqueología de la Arquitectura, 4, pp. 11-19.

Moretti, I. e Stopani, R. 1974: Architettura romanica religiosa nel contado fiorentino, Firenze.

Parenti, R. 1987: Una proposta di classificazione tipologica delle murature postclassiche, in G. Biscontin e R. Angeletti (a cura di), "Conoscenze e sviluppi teorici per la conservazione di sistemi tradizionali in muratura", Atti del Convegno di Studi (Bressanone 23-26 giugno 1987), Bressanone, pp. 49-61.

Parenti, R. e Quiros Castillo, J. A. 2000: La produzione dei mattoni della Toscana medievale (XII-XVI secolo). Un tentativo di sintesi, in P. Boucheron, Y. Thebert e H. Broise, "La brique antique et médiévale : production et commercialisation d'un materiau", Atti del Colloquio Internazionale di Studi, Parigi, 16-18 novembre 1995, Roma, pp. 1-17.

Pierotti, P. 2003: Manuale di Sismografia Storica. Lunigiana e Garfagnana, Pisa.

Pierotti, P. e Ulivieri, D. 2001: Culture sismiche locali, Pisa.

Pittaluga, D. e Quiros Castillo, J. A. 1997: Mensiocronologie dei laterizi della Liguria e della Toscana: due esperienze a confronto, in "I Congresso Nazionale di Archeologia Medievale”, Pisa, 29-31 maggio 1997, Firenze, pp. 460-463.

Quiros Castillo, J. A. 1997: La mensiocronologia dei laterizi della Toscana: problematiche e prospettive di ricerca, in "Archeologia dell'Architettura", II, Firenze, pp. 159-166.

Quiros Castillo, J. A. 2001: Mensiocronologia e produzione di laterizi in Toscana in età medievale, in "I laterizi in età medievale. Dalla produzione al cantiere", Atti del Convegno Nazionale di Studi, Roma, 4-5 giugno 1998, Roma, pp. 31-44.

Salvestrini, F. 2011: Eremitismo - Cenobitismo. La realtà di Santa Maria di Vallombrosa in età medievale, in S. Bertocci e S. Parrinello (a cura di), "Architettura eremitica. Sistemi progettuali e paesaggi culturali", Atti del Secondo Convegno Internazionale di Studi, Vallombrosa, 24-25 settembre 2011, Firenze, pp. 33-39. 